matrix to the cerebral cortex and are consistent with a neurodevelopmental cause of developmental dysphasia. (Cohen $M$ et al. Neuropathological abnormalities in developmental dysphasia. Ann Neurol June 1989; 25: 567-570).

COMMENT. The Child Neurology Society Task Force on the nosology of disorders of higher cerebral function in children (1981) defined developmental dysphasia as a delayed, and usually aberrant, acquisition of language for cammunicative use, provided the delay is not accounted for by deafness or by severe mental retardation. It must be distinguished from acquired aphasia as a result of focal pathological lesions usually affecting the left hemisphere. In the present case report there was no evidence of an acquired insult or disease process although the patient did have a delay in walking. The language disorder was associated with oromotor apraxia, anomia and the use of gesture. The assumed developmental basis for the dysphasia was correlated with subtle developmental brain anomalies characterized by symmetry of the plana temporale similar to that reported in cases of dyslexia. (Galaburda AM et al. Ann Neurol 1985; 18:222).

\title{
PSEUDOBULBAR PALSY AND MACROGYRIA
}

Four patients with medically intractable epilepsy, pseudobulbar palsy and mental retardation and found to have bilateral central macrogyria on CT and MRI are reported from the Department of Neurology and Neurosurgery, McGill University, and the Montreal Neurological Institute and Hospital, Montreal, Canada; and the Department of Neurology, University of Minnesota, St. Paul Ramsey Hospital, St. Paul, MN. The pseudobulbar palsy was associated with oromotor incoordination, developmental delay and mild retardation. Minor seizures developed between the ages of eight and nine years and one patient had infantile spasms at three months of age. Electroencephalographic epileptogenic abnormalities were secondary generalized or multifocal. CT scans revealed symetrical bilateral sylvian and rolandic macrogyria extending into the parietal regions. The cortex appeared thick and smooth with the underlying white matter diminished. The MRI confirmed the CT findings and showed that the abnormal cortex had a lower signal as compared to normal gray matter of frontal and occipital regions. The thick cortical structures surrounded a large central sulcus reminiscent of a fetal sylvian fissure. Two patients tried on multiple anticonvulsants continued to have frequent seizures whereas two treated by callosotomy had no subsequent drop at tacks and improved behavior. The authors suggest that the clinical and imaging features of these patients indicate a distinct and specific syndrome and the malformations appear to result from specific derangement of neuronal migration. (Kuzniecky R, Andermann F et al. Bilateral central macrogyria: Epilepsy, pseudobulbar palsy, and mental retardation -- A recognizable neuronal migration disorder. Ann Neurol June 1989; 25:547-554).

COMENT. Neuronal migration disorders, including agyria (or lissencephaly), macrogyria (or pachygyria), polymicrogyria, 
schizencephaly, and other heterotopias of the gray matter are being recognized more frequently by the use of the magnetic resonance imaging technique. The role of neurosurgical treatment in patients with neuronal migration disorders is being explored. The early recognition and effective surgical management of these cases might improve prognosis and prevent the development of intractable epilepsy and mental deterioration.

\section{DEVELOPMENTAL DYSPHASIA SUBTYPES}

The syndrames of developmental dysphasia and their remediation are outlined in the selected proceedings of the Fourth International Child Neurology Congress held in Jerusalem, Israel March 16-20, 1986. Six dysphasic syndromes are identified through the cambined psycholinguistic/ aphasiology model: 1) Verbal dyspraxia, 2) phonological production deficit, 3) verbal auditory agnosia, 4) phonologic-syntactic deficit, 5) lexical-syntactic deficit, and 6) semantic pragmatic deficit. In subtypes 1 and 2 impairment is in the expressive system with comprehension relatively intact. Verbal dyspraxia is a severe expressive disorder in which the child is extremely nonfluent and may be mute. Phonological production deficit is characterized by fluency but nonetheless unintelligibility. In both syndromes the production of consonants is more difficult than of vowels. Apraxia of speech and developmental misarticulation are terms sometimes applied to these expressive dysphasias. All dyspraxic dysphasias are difficult to treat and many require speech and language therapy beyond the preschool years. Many need to be introduced to a visual-manual system such as reading and writing at an early age, and some severe cases require formal sign language in conjunction with verbalization. Subtype 3 verbal auditory agnosia, camonly called word deafness, is an inability to decode spoken language at the level of phonology. Despite normal peripheral hearing the individual is unable to derive any meaning from the sounds he or she hears. These children comprehend virtually nothing and are essentially mute. Naming an object, drawing a picture of the object, and naming the picture with a printed label facilitates vocabulary building and the labels can then be used to make requests. Subtype 4 is a mixed receptive expressive deficit and results in telegraphic speech with amission of prepositions, verbs, and the endings on nouns and verbs. The use of gestures, cammication boards and written words to supplement oral language are encouraged. In dysphasia subtype 5, lexical syntactic deficit, syntax or the arrangement of words is immature and comprehension of abstract language may be poor. Language is dysfluent with multiple hesitations, false starts and self-corrections. The syndrome is seen in both autistic and nonautistic children. Dysphasia subtype 6, semantic pragmatic deficits with hyperverbal or loquacious speech but their commumicative intent and semantic content are limited and superficial. Jargon, neologisms, paraphasias, and circumlocutions are camon. Speech is quite rapid and "cluttered". The subtyping of dysphasic children permits the design of specific interventions for the particular language needs of the children in each group. (Allen DA, Mendelson L, Rapin, I. Syndrame specific remediation in preschool developmental dysphasia in Child Neurology and Developmental Disabilities, edited by French, JH, Harel, S, Casaer, P. Baltimore, London, Sydney, Toronto. Paul H. Brookes Publishing Campany, 1989). 